possible without the earlier support of the Foundation for Dr. Lee's work.

\section{NOTATION}

$c^{\prime} \quad=$ root-mean-square concentration fluctuation

$I_{s} \quad=$ intensity of segregation

$L_{s} \quad=$ scale of mixing

$N_{\text {Sc }}=$ Schmidt number $(\nu / D)$

$r \quad=$ radius

$r_{\mathrm{o}} \quad=$ pipe radius

$t \quad=$ time

$\vec{U} \quad=$ local mean velocity

$u^{\prime}=$ root-mean-square axial Eulerian velocity fluctuation

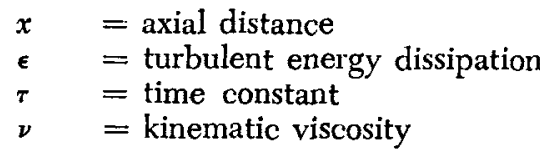

\section{LITERATURE CITED}

I. Lee, Jon, and R. S. Brodkey, A.I.Ch.E. J., 10, 187 (1964).

2. Brodkey, R. S., ibid., 12, 403 (1966).

3. Lee, Jon, Ph.D. thesis, Ohio State Univ., Columbus (1962).

4. Gegner, J. P., M.S, thesis, Ohio State Univ., Columbus (1965).

5. Corrsin, Stanley, A.I.Ch.E. J., 3, 329 (1957); 10, 870 (1964).

6. Knox, J. S., M.S. thesis, Ohio State Univ., Columbus (1966).

\title{
Accuracy in Residence Time Measurements
}

\author{
R. L. CURL and M. L. MeMILLAN \\ University of Michigan, Ann Arbor, Michigon
}

Residence time distributions in flow or reaction systems give information about internal flow characteristics and are useful for predicting conversions with first-order reactions or in completely segregated reactors. The mean residence time provides a way to measure system volume by external means and the variance has found wide utility in estimating dispersion parameters in system models (1).

It is, of course, not possible to make these measurements with absolute precision. In particular, every real system has internal life expectancies extending to infinity, so that analytical problems or impatience eventually truncates all measurements.

The usual technique is to inject a pulse of tracer material into the system and then to follow the effluent time-dependent concentration. Alternatively, tests in the form of saturation or elution steps may be used. If the system is linear these responses are theoretically interchangeable by well-known techniques. If $c(t)$ is the effluent concentration response to a true pulse input, the normalized residence time density distribution $f(t)$ is estimated, in practice, from

$$
\begin{aligned}
f^{*}(t) & =\frac{c(t)}{\int_{0}^{t^{*}} c(t) d t} & & 0 \leq t<t^{*} \\
& =0 & & t \supseteq t^{*}
\end{aligned}
$$

The time $t^{\circ}$ is the last time at which the tracer may be detected or when, for analytical reasons, it appears that the response has gone to zero. The limit $t^{\circ} \rightarrow \infty$, if it were possible to obtain, gives the true distribution $f(t)$. As a check on the accuracy of the measurement, the material balance

$$
\frac{q}{Q} \doteq \int_{0}^{t^{*}} c(t) d t
$$

M. L. McMillan is with General Motors Research Laboratory, Warren, Michigan. is often used. Equation (2) is an equality only in the limit $t^{*} \rightarrow \infty$. One question to be considered here is the significance of the error caused by having finite $t^{\circ}$.

From the estimated $f^{*}(t)$ the estimated (cumulative) residence time distribution $F^{*}(t)$ is obtained from

$$
F^{*}(t)=\int_{0}^{t^{*}} f^{*}(t) d t
$$

For a linear system this is equivalent to the response to a saturation step, while $\left(1-F^{*}(t)\right)$ would be the response to an elution step. The value of $t^{*}$ may differ in the three techniques (pulse, saturation step, and elution step), depending on analytical accuracy. This is usually inherently greatest in elution step tests, as the concentration of a tail need only be distinguished from zero. A pulse test attenuates rapidly unless the input pulse is very great, and a saturation step approaches a final, relatively large concentration.

The mean residence time in a system may be estimated from

$$
\overline{t^{*}}=\int_{0}^{t^{*}} t f^{*}(t) d t=\int_{0}^{1} t d F^{*}
$$

and the second moment, from which the variance is obtainable, from

$$
\bar{t}^{2 *}=\int_{0}^{t^{*}} t^{2} f^{*}(t) d t=\int_{0}^{1} t^{2} d F^{*}
$$

The limit $t^{*} \rightarrow \infty$ gives, in all of the above cases, the fundamental relations between true values of $f(t), F(t)$, $\bar{t}$, and $\bar{t}^{2}$. They are written in terms of $t^{*}$ to emphasize the nature of the approximations we are forced to use.

A series of measurements were reported by Lapidus (2) and Schiesser and Lapidus (4) in which step and pulse tests through beds packed with both nonporous and porous spheres were used to obtain the holdup in the bed during trickle phase operation. They found agreement between pulse and step tests when determining 
mean residence time in beds of nonporous spheres, but with porous spheres the values obtained from Equation (4) differed greatly. $F^{*}(t)$ also differed greatly for the two types of tests. However within analytical accuracy the material balance [Equation (2)] always checked out to better than $98 \%$. Numerous tests excluded nonlinear effects. An explanation was suggested but in a truly linear system no explanation is possible if it is assumed that all tests had been conducted with sufficient accuracy. Rothfeld and Ralph (3), however, reported agreement between pulse and step tests in a trickle phase bed. A confirmation of their result is presented here, as well as a possible explanation of the contradiction found by Schiesser and Lapidus (4). An analysis is presented to show that a very significant error may be caused by truncating experiments at too low values of $t^{*}$ and that a material balance check [Equation (2)] may be completely misleading.

\section{EXPERIMENTAL}

A glass column, $3 \mathrm{~cm}$. I.D., $38 \mathrm{~cm}$. high, was randomly packed with $1 / 8$-in. Harshaw Alundum catalyst pellets. Short sections at both ends packed with glass beads helped to distribute the flow. The column was operated liquid-filled for convenience, as the trickle phase nature of the previous work is not at issue in discrepancies between pulse and step tests. Flow was provided by constant-head gravity feed and was metered with rotameters. All experiments were performed at a volumetric flow rate of 2.0 liters $/ \mathrm{min}$. ( $\pm 2 \%$ )

One reservoir provided distilled water for pulse and elution tests. Another provided 0.05 to 0.20 normal sodium chloride solution for saturation tests. The flows could be switched quickly between the reservoirs for step tests. For pulse tests 2 to $3 \mathrm{ml}$. of sodium chloride tracer, between 2.0 and 3.0 normal, were injected rapidly into a stream of distilled water flowing through the column.

Before pulse or saturation tests the column was flushed with distilled water for a period of 2 to $8 \mathrm{hr}$.; sodium chloride solution was used for elution tests. No differences could be detected between feed and effluent concentrations when a test began.

Samples of effluent were taken at measured time intervals and their electrical conductivity was determined at $34.5 \pm$ $0.5^{\circ} \mathrm{C}$. with an Industrial Instruments $\mathrm{RC}-1 \mathrm{~B}$ conductivity bridge. A conductivity-concentration calibration was obtained. With extreme care to avoid contamination of dilute solutions, concentration measurements were accurate to $\pm 1 \%$ over the range from 0.3 to $2 \times 10^{-5}$ normal. A correction was applied for the inherent conductivity of the distilled water used.

The duration of each run was about $50 \mathrm{~min}$. (the saturation runs a bit shorter).

\section{RESULTS}

The experimental concentration data from pulse tests are converted to the normalized forms $f^{*}(t)$ and $F^{*}(t)$ by means of Equations (1) and (3). All comparisons were made on the basis of $1-F^{*}(t)$. For saturation and elution tests this was obtained directly as $c(t) / c_{o}$ and $1-c(t) / c_{o}$, respectively. The results are compared in Figure 1. There is no significant difference between the results from pulse, saturation step, and elution step, except near the termination of the experiments.

The elution step has the greatest accuracy as it could be followed for the longest time. It implies that at about 2,800 sec., only 0.0003 of the original tracer remained in the bed. From the relation between step and pulse tests, this is also the true material balance for the pulse tests. Therefore the effective material balance was $99.97 \%$. The measured material balance [Equation (2)] was $100.7 \%$ for a pulse test, but this is subject to the errors of concentration measurement, flow rate variation, injected pulse concentration, and volume and integration errors, and should not be expected to be better than \pm



Fig. 1. Comparison of results of pulse, elution step, and saturation step tracer tests on a column packed with porous pellets.

$5 \%$. In these experiments the measured material balance is at best a crude estimate of the value obtained from an elution test. We shall see that measured material balances in pulse tests may be no measure of the accuracy of the means or variances of residence times.

Using Equation (4) we may obtain an estimate of the void volume of the bed (including pore volume). As void fraction these were found to be $0.56,0.59$, and 0.56 from the pulse, elution step, and saturation step, respectively. The bed void volume determined by a weighing technique gave $0.62 \pm 10 \%$. These are in satisfactory agreement.

By fitting an exponential-decay equation to the later points of the measured responses, it is possible to estimate the effect of carrying the integrations in Equations (2), (3), and (4) to infinite time. Correction of the response curves $\left[1-F^{*}(t)\right]$ and mean residence times in this manner caused less than $2 \%$ change. We conclude that the tests had been carried effectively to infinite time at $t^{*}=2,800 \mathrm{sec}$.

\section{ERROR MODEL}

Assume an idealization of the pulse response shown in Figure $2 a$ to be that shown in Figure $2 b$. The material that passes through the bed without diffusing into particles is represented as an impulse of area $1-u$ located at time $a$. The subsequent exponential decay, if taken to infinity, has area $u$. This form is plotted in Figure $2 c$ as $1-F(t)$. The resemblance to measured responses is evident. The equation for the idealized pulse response is then

$$
\begin{aligned}
f(t) & =0 \quad(0 \leq t<a) \\
& =(1-u) \delta(t-a)+u \omega e^{-\omega(t-a)} \quad(a \leq t)
\end{aligned}
$$

Now if $t^{*}$ is the time at which a pulse experiment is 

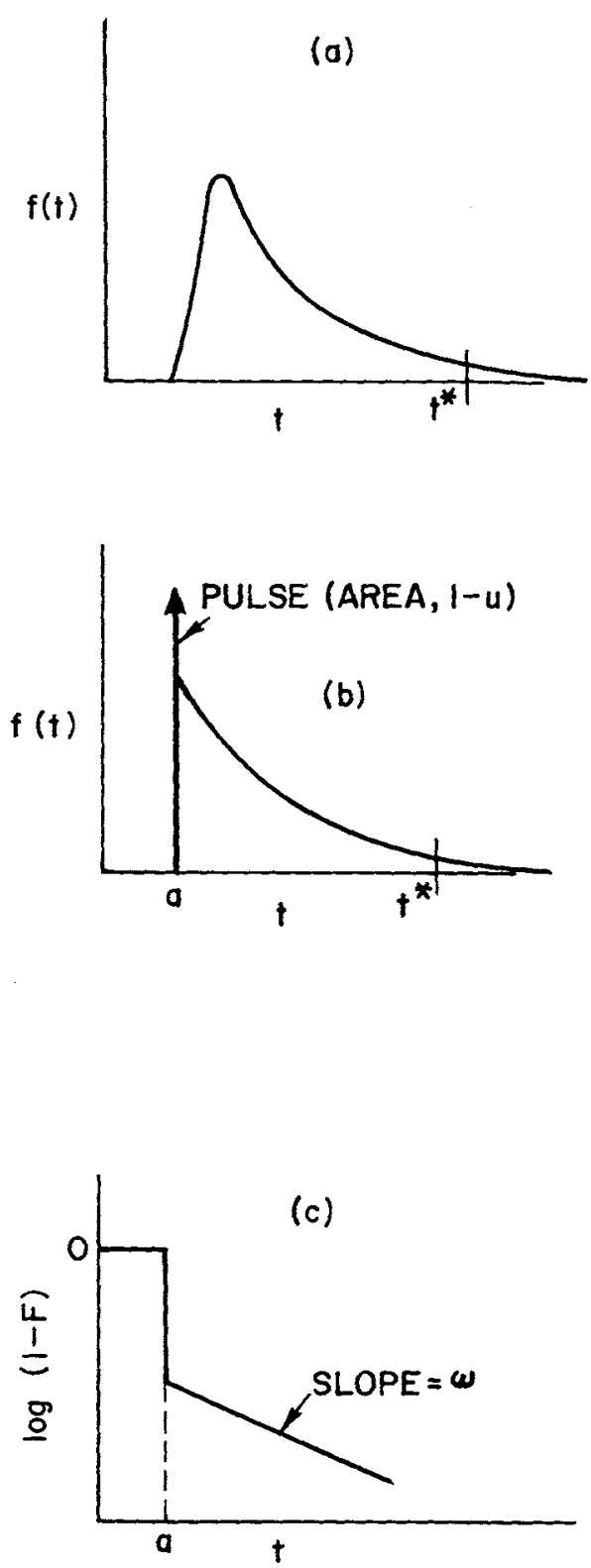

Fig. 2 (a) Possible pulse response of a system. (b) Idealization of actual response as the sum of a pulse and exponential decay. (c) Idealized response in semilogarithmic coordinates.

terminated due to further undetectability of the tracer, the material balance error would be

$$
\epsilon=\int_{t^{*}}^{\infty} u \omega e^{-\omega(t-\alpha)} d t
$$

which gives the relation between $t^{*}$ and $\epsilon$ :

$$
\omega t^{*}=\omega a+\ln \frac{u}{\epsilon}
$$

The estimated pulse response, which has been truncated at $t^{*}$, is then

$$
\begin{aligned}
f^{*}(t) & =\frac{f(t)}{1-t} & & \left(0 \leq t<t^{*}\right) \\
& =0 & & \left(t \geq t^{*}\right)
\end{aligned}
$$

Evaluating Equation (4) for this truncated distribution based on Equation (6) and dividing by the true $\bar{t}$ of Equation (6), we obtain the ratio $\bar{t}^{*}, \bar{t}$, a measure of the error caused by truncating the true pulse response. Car- rying out the necessary integration and using Equation (7), we obtain

$$
\gamma_{1 p}=\frac{\bar{t}^{\circ}}{\bar{t}}=\frac{1}{(1-\epsilon)}\left[1-\epsilon\left(\frac{a \omega+1+\ln \frac{u}{\epsilon}}{a_{\omega}+u}\right)\right]
$$

This is for a constant flow rate, the ratio of holdup that would be found experimentally to the true holdup for a pulse test.

Similarly, from Equation (5) for the second moment, we obtain

$$
\begin{aligned}
\gamma_{2 p}=\frac{\bar{t}^{2}}{\overline{t^{2}}}= & \frac{1}{(1-\epsilon)} \\
& {\left[\begin{array}{l}
\left.1-\epsilon\left(\frac{\left(\ln \frac{u}{\epsilon}+a \omega+1\right)^{2}+1}{(a \omega)^{2}+2 u(a \omega+1)}\right)\right)
\end{array}\right.}
\end{aligned}
$$

The same type of analysis may be applied to step tests. The step response equivalent to Equation (6) is

$$
\begin{aligned}
F(t) & =0 & & (0 \leqslant t<a) \\
& =1-u e^{-\omega(t-a)} & & (a \leqslant t)
\end{aligned}
$$

In an actual experiment the value of $c(t) / c_{o}$ appears to reach zero or one (for elution and saturation step, respectively) at some time $t^{*}$, which bears the same relation to $\epsilon$ as before. Then the truncated distribution $F^{*}(t)$ is

$$
\begin{aligned}
F^{*}(t) & =0 & & (0 \leq t<a) \\
& =1-u e^{-\omega(t-a)} & & \left(a \leq t<t^{*}\right) \\
& =1.0 & & \left(t^{*} \leq t\right)
\end{aligned}
$$

No renormalization need be applied in this case. Evaluating Equation (4) for this truncated distribution and dividing by the true $\bar{t}$, which is the same as before, we obtain

$$
\gamma_{1 s}=1-\frac{\epsilon}{a_{\omega}+u}
$$

and, for the similar ratio of second moments

$$
\gamma_{2 s}=1-\frac{2 \epsilon\left(1+a \omega+\ln \frac{u}{\epsilon}\right)}{(a \omega)^{2}+2 a \omega u+2 u}
$$

It is evident, by comparing Equations (10) and (14) or (11) and (15), that $\gamma_{1 s}>\gamma_{1 p}$ and $\gamma_{2 s}>\gamma_{2 p}$. Step tests of whatever sort are more accurate than pulse tests even with the same material balance failure. A saturation step may, however, be less accurate than an elution test as it is more difficult to follow the tail of a saturation test analytically, that is, to distinguish small differences in large concentrations.

The data reported here, some of that obtained by Schiesser (6) [reported by Schiesser and Lapidus (4) in their Figure 10] and a set obtained by Rothfeld and Ralph (3), have been tested by means of the above relations. In each case the values of $a, u$, and $\omega$ were estimated from the measured elution step responses. The value of $t^{*}$ for any particular case is the time at which the last data point is obtained. The corresponding $\bullet$ is the value from the elution step data at $t^{*}$ (this being the best measure of the true value). Comparison of predicted values of $\gamma_{1 p}, \gamma_{2 p}, \gamma_{1 s}$, and $\gamma_{2 s}$ (for both elution and saturation steps) are shown in Table 1.

The elution test used by Schiesser appears satisfactory for estimating $\bar{t}$, but the pulse and saturation tests give $\bar{t}$ values which are too low. The conditions of the Rothfeld 
Test

a, sec.

$u$

Pulse

Sat. step

Elut. step

Schiesser (5)

Pulse

Sat. step

Elut. step

Rothfeld and Ralph (3)

Pulse

Steps
30

0.012

60
0.00555

and Ralph experiments appear satisfactory for estimating both $\bar{t}$ and $\bar{t}^{2}$. There is a reasonable correspondence between the predicted $\gamma_{1}$, and those found experimentally by Schiesser (5). This suggests that their discrepancies between the results of pulse, saturation step, and elution step tests arise primarily from the experimental truncation of the tests due to analytical limitations. As far as a material balance check is concerned, we see that with certain parameters for a pulse test, a $1 \%$ error in material balance can cause a $50 \%$ (or more) error in holdup measurements, illustrating the extreme importance of the tails in estimates derived from residence time measurements. At best, material balance checks cannot be much better than $1 \%$. In porous beds the necessary precision of closure of the material balance is orders of magnitude greater than can be determined directly.

If an experiment is poor for mean residence time determination, it is worse for determining the variance of residence times. For example, if Equation (11) for the second moment error is applied to the pulse test data represented in Figure 1, with the same parameters as above, $\gamma_{2 p}=0.70$. Even these pulse data are inadequate

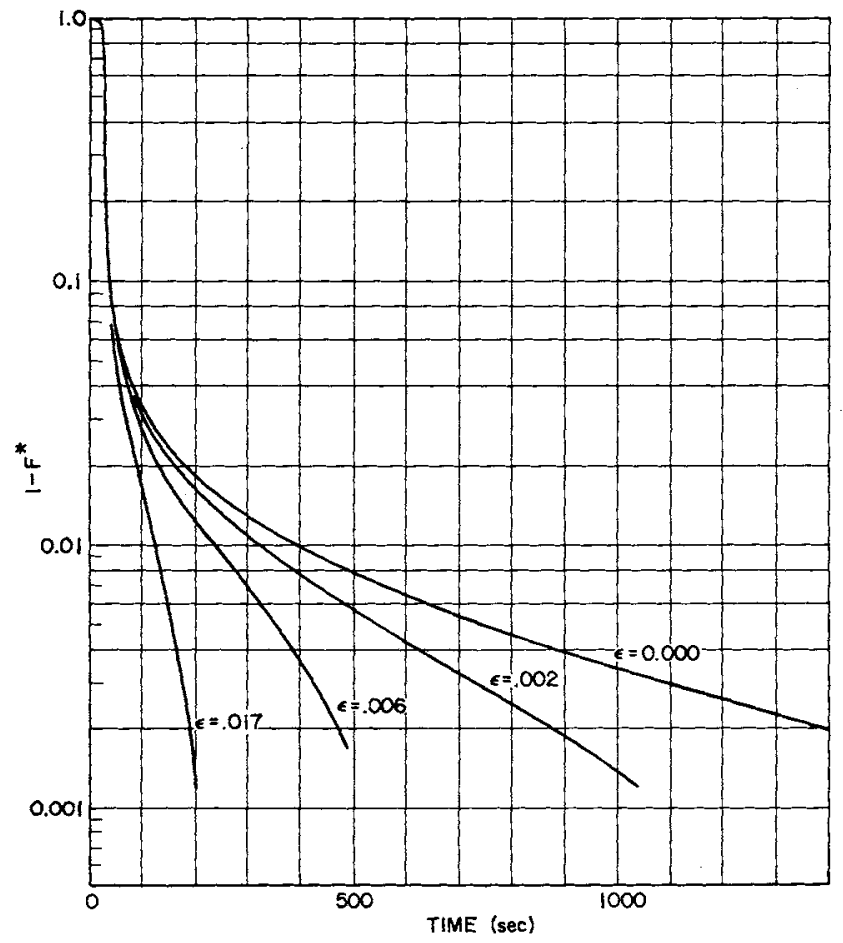

Fig. 3. The effect on a pulse response $(\epsilon=0)$ produced by the artificial introduction of various material balance errors through truncation. $\boldsymbol{\epsilon}$

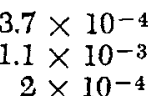

$\gamma_{1} \quad \gamma_{1}(\exp$.

$\gamma 2$

$1.1 \times 10^{-3}$
$2 \times 10^{-4}$

0.967

0.979

0.996

0.700

0.720

0.920

0.089
0.034
0.009

0.540

0.890

0.970

0.59

0.73

0.07

0.53

0.81

0.003

0.978

0.920

0.003

0.996

0.975

for determining variance. The elution data might be satisfactory. Higher moments are nearly inaccessible by experiment unless truly extreme care is taken.

As one final demonstration of the effect of small material balance failure, the data in Figure 1 are replotted in Figure 3 by assuming various artificial values for $t^{*}$ or equivalently of material balance failures. That is, the true pulse response was obtained by differentiating the elution data, by truncating, and then by renormalizing, on the basis of Equation (1), before recalculating $F^{*}(t)$. We see that an artificial $99.8 \%$ balance gives considerable error in $1-F^{*}(t)$ and that a $99 \%$ balance is useless. The departures of these curves resemble the respective elution, saturation, and pulse curves reported by Schiesser and Lapidus (4).

Equations (10), (11), (14), and (15) could be used in reverse to determine the precision necessary (the value of $\epsilon$ or $t^{*}$ ) for an accurate estimate of mean residence times or variances from pulse tests. However, an elution test would be desirable for estimating the parameters necessary for a prediction of possible errors in itself, or in pulse or saturation tests.

\section{NOTATION}

$a \quad=$ pulse delay in response model, sec.

$c(t)=$ measured concentration response

$c_{o}=$ initial (final) concentration in elution (saturation) step tests

$f(t)=$ residence time density distribution

$F(t)=$ residence time distribution

$q=$ moles injected in pulse tests

$Q \quad=$ volumetric flow rate

$t=$ time, sec.

$t^{*}=$ test truncation time, sec.

$u=$ response model parameter

$\gamma_{n}=$ ratio of estimated to true $n^{\text {th }}$ moment of model response $(n=1,2, \ldots)$

$\epsilon=$ material balance failure in response model

$\omega=$ decay frequency in response model, sec. ${ }^{-1}$

\section{Superscripts}

- $\quad$ mean value

- $\quad=$ truncated responses

\section{LITERATURE CITED}

1. Levenspiel, O., "Chemical Reaction Engineering," Wiley, New York (1962).

2. Lapidus, Leon, Ind. Eng. Chem., 49, 1000-1006 (1957).

3. Rothfeld, L. B., and J. L. Ralph, A.I.Ch.E. J., 9, 852-859 (1963).

4. Schiesser, W. E., and Leon Lapidus, ibid., 7, 163-171 (1961).

5. Schiesser, W. E., Ph.D. thesis, Princeton Univ., N. J. (1960). 